Meta

Journal des traducteurs

Translators' Journal

\title{
Pour une dimension culturelle de la traduction juridique
}

\section{Michel Sparer}

Volume 24, numéro 1, mars 1979

La traduction juridique

URI : https://id.erudit.org/iderudit/004204ar

DOI : https://doi.org/10.7202/004204ar

Aller au sommaire du numéro

Éditeur(s)

Les Presses de l'Université de Montréal

ISSN

0026-0452 (imprimé)

1492-1421 (numérique)

Découvrir la revue

Citer cet article

Sparer, M. (1979). Pour une dimension culturelle de la traduction juridique.

Meta, 24(1), 68-94. https://doi.org/10.7202/004204ar d'utilisation que vous pouvez consulter en ligne.

https://apropos.erudit.org/fr/usagers/politique-dutilisation/ 


\section{Pour une dimension culturelle de la traduction juridique}

Il est temps que la traduction juridique se désolidarise de la mentalité de ghetto extra-culturel dans laquelle la profession juridique s'enferme encore trop souvent. Nous nous sommes débarrassés depuis peu de la fidélité littérale pour adopter avec profit une conception plus affinée et plus autonome du rôle du traducteur, celle qui consiste à traduire l'idée avant de s'attacher au mot.

J'ose prétendre qu'il est possible et souhaitable d'aller plus loin, d'aller vers une dimension culturelle de la traduction. Il ne s'agirait plus de passer d'un mot à l'autre, ni de faire passer un même ensemble d'idées d'une langue à une autre. Imaginons que la priorité du traducteur soit enfin de servir le plus efficacement possible le but fixé au texte d'arrivée. Il faut pour cela mettre la finalité du texte d'arrivée en première place dans les critères de détermination des moyens à prendre pour produire en fin de compte le même effet sur les lecteurs du texte de départ et sur ceux du texte d'arrivée. Cela requiert une sensibilité culturelle à laquelle prépare particulièrement bien le métier de traducteur. Cela demande notamment une connaissance des réflexes culturels et des leviers propres à les provoquer.

Le texte juridique, telle la loi, a une caractéristique qui explique en grande partie les supplices qu'il fait souvent subir à la langue. L'usager de ce texte, le justiciable, vous et moi, doit respecter la règle qu'il porte. Certaines apparences laisseraient croire que le rédacteur de la loi ou du règlement, se fondant sur l'obligation de respecter les normes portées par le texte, abandonne au justiciable le fardeau de les y « découvrir ». Tel n'est pas exactement le cas. En effet, le rédacteur est accablé par de nombreuses contraintes, dont celle d'un échéancier souvent irréaliste. Par conséquent, il ne peut pas toujours accorder l'attention qu'il voudrait à un aspect primordial : l'intelligence par le plus grand nombre.

D'où la facilité de laisser glisser la langue du droit vers ce que je me permettrai d'appeler une situation \& hors culture », pour faire, qu'on ne m'en tienne pas rigueur, un mauvais néologisme. Or, je crois que l'auteur de la norme sociale de base qu'est devenue la loi a quand même intérêt à ce que cette norme ne soit pas culturellement étrangère aux justiciables ; ni dans les principes qui la soustendent, ni dans son expression. Il est connu qu'une société ne peut s'organiser ni se maintenir harmonieusement sans que les normes dont on la charpente soient 
un tant soit peu acceptées. Je crois qu'elles doivent donc être « en phase avec les réflexes culturels fondamentaux.

De là à penser qu'en traduisant des lois ou des règlements, on «importe * un type d'organisation sociale, il n'y a qu'un pas que je m'empresse de franchir.

Sans être isolationniste, je prétends qu'il est dangereux de traduire des règles sans tenir compte du fait qu'elles ont été conçues et rédigées par et pour d'autres personnes, en l'occurrence les anglophones, qui pensent et vivent différemment de nous. Le traducteur ne devrait-il pas, en conséquence, s'intéresser en premier lieu aux aspects téléologiques de la traduction ? Ce qui consisterait, à mon sens, à tenir compte en priorité de l'impact qu'on attend du texte d'arrivée et ensuite, dans la mesure où cela est souhaitable et possible, des moyens que l'auteur a pris pour créer cet impact sur la communauté visée par le texte de départ.

Pour l'instant, voyons d'une manière plus pratique quelques aspects de la traduction juridique tels que j’ai pu les observer.

\section{INTRODUCTION}

Parmi les préoccupations que soulèvent, d'une part, la nécessité de traduire des textes juridiques et, d'autre part, l'activité de traduction elle-même, certaines, il me semble, sont trop souvent négligées, voire ignorées. Par exemple, avant de traduire ou de faire traduire un texte juridique, trop rares sont ceux qui se demandent s'il est possible de produire dans la langue d'arrivée un texte qui ait rigoureusement le même sens immédiat et surtout la même portée.

Une autre préoccupation qui est heureusement un peu plus répandue consiste à se demander comment un juriste qui a comme langue maternelle la langue d'arrivée aurait rédigé le texte, si on lui avait demandé de le créer.

C'est autour de ces deux axes que tourneront mes propos, placés sous le double éclairage de la science juridique et de la psycho-linguistique. Précisons, d'entrée, que si je me hasarde sur le terrain linguistique ou psycho-linguistique, ma formation académique ne m'y a pas préparé. Je ne veux en cela que livrer les quelques réflexions qui me sont venues en tant que simple usager de la langue, versé depuis quelques années dans l'étude de la rédaction des lois et de la traduction juridique.

Bien souvent, l'intervention du juriste en matière de traduction juridique a pour effet de faire prévaloir les impératifs du droit sur ceux du style, de la grammaire ou de la terminologie. En tant que juriste, je vous propose d'aborder les idées que nous avons évoquées plus haut sous l'angle d'une coopération entre les deux disciplines, pour une fois remises à leur place, c'est-à-dire sur le même plan. Une coopération ne peut produire ses effets utiles que si les protagonistes agissent chacun en pleine connaissance des contraintes de l'autre. Pour cela, après avoir proposé quelques définitions générales et rappelé quelles sont les sources du droit, nous examinerons comparativement les modes de formation et de formulation respectifs du système juridique de type britannique, et du système juridique de type romano-germanique (communément appelé droit français), et enfin, les 
conséquences directes de la dualité intellectuelle et formelle du droit canadien et québécois sur la formulation des textes juridiques que vous avez à traduire. Nous raccrocherons à tout cela quelques considérations sur les rapports de travail entre les traducteurs et les juristes.

\section{QUELQUES NOTIONS SUR LA FORMATION DE LA RÈGLE DE DROIT}

Je vous propose, pour fixer ou rafraîchir des connaissances académiques ou intuitives que vous pouvez avoir des principaux concepts du droit, quelques définitions dont je tiens à souligner, d'une part, qu'elles ne sont pas nécessairement les seules valables pour les concepts concernés et, d'autre part, qu'en l'absence de dictionnaire de droit au Québec, en langue française, il est inévitable d'avoir à se reporter à des dictionnaires ou lexiques conçus en général dans d'autres pays francophones, notamment la France. Les dictionnaires ou lexiques français, étant faits en France, sont orientés vers le droit français. C'est pourquoi je me contenterai de proposer en définition des termes qui, par leur généralité, désignent des concepts semblables dans tous les pays organisés.

\section{A. Définitions générales}

Droit : le droit est l'ensemble des règles régissant la vie en société et sanctionnées par la puissance publique.

Le mot droit que nous avons défini ici dans son sens de droit objectif peut également désigner le droit subjectif qui est, lui, une prérogative attribuée à un individu, dans son intérêt, lui permettant de jouir d'une valeur ou d'exiger d'autrui une prestation. C'est toute la différence entre LE droit, pris en tant que système, et LES droits que chacun revendique, comme le droit de propriété, le droit d'association, ou encore le droit de vivre en paix.

Droit public : c'est l'ensemble des règles régissant les rapports entre la puissance publique et les particuliers.

Droit privé : c'est l'ensemble des règles régissant les rapports entre particuliers. Soulignons que le droit privé peut également servir à régir les rapports entre l'État et les particuliers, lorsque l'État se comporte comme une personne de droit privé, notamment lorsqu'il passe des contrats ordinaires comme le contrat de travail ou le contrat de vente, par exemple.

Droit commun : c'est l'ensemble des règles applicables en droit privé. On considère d'ordinaire que le droit civil constitue le droit commun.

\section{B. Hiérarchie des textes juridiques}

Je vous propose de classer les textes juridiques en deux catégories : les textes à caractère législatif et les textes non législatifs.

C'est parmi les textes à caractère législatif qu'il est utile de rappeler qu'il existe une hiérarchie, non pas fondée sur le critère de la valeur obligatoire, mais sur l'obligation qu'ont certains textes de respecter ou d'être conformes à certains 
autres textes. Dans la hiérarchie des textes législatifs, nous pouvons distinguer deux catégories : 1) la législation au sens strict, 2) la législation dite déléguée.

\section{La législation au sens strict}

a) la Constitution : c'est l'ensemble des règles écrites ou coutumières qui déterminent la forme de l'État, la dévolution et l'exercice du pouvoir.

La Constitution détermine la forme de l'État en précisant si l'État est par exemple une république, une monarchie ou encore une dictature. Elle détermine d'autre part la dévolution des pouvoirs, c'est-à-dire à qui et comment sont dévolus les pouvoirs de l'Etat, et enfin l'exercice du pouvoir, c'est-à-dire comment les pouvoirs de l'État doivent être exercés par ceux à qui ils sont dévolus.

b) la Loi : c'est une règle écrite générale et permanente élaborée par le Parlement.

En effet, la loi est écrite. Elle est générale, ce qui signifie qu'elle s'impose à tous. Elle est permanente, ce qui signifie qu'en principe, elle s'impose telle qu'elle est, tant que le Parlement n'a pas décidé de la modifier, de la remplacer ou de l'abroger. Enfin, elle est élaborée par le Parlement, ce qui signifie que le Parlement est la seule entité dans l'État qui ait compétence pour l'élaborer. Il est donc hasardeux sinon inexact de parler, par exemple, de loi lorsqu'il s'agit d'une règle établie par une municipalité ou par une entreprise.

\section{La législation dite déléguée}

Il arrive que le Parlement, le législateur, estime opportun, pour des raisons diverses, de se limiter à déterminer dans la loi de grands principes; il délègue au gouvernement, qu'on désigne parfois par l'expression « pouvoir exécutif », le pouvoir de préciser la loi quant à sa portée et à ses modalités d'application. Il se contente alors de dresser dans la loi les grandes lignes de la politique, en laissant le détail d'application au soin du gouvernement. Le gouvernement, alors récipiendaire d'un pouvoir de législation, produit des textes qu'on appelle règlement, et qui ont valeur de loi, c'est-à-dire qu'ils s'imposent à tous.

On peut donc définir le règlement comme un acte impersonnel et de portée générale édicté par les autorités exécutives compétentes. Notons qu'au Canada, au Québec, dans tous les pays de droit britannique, le gouvernement doit, pour pouvoir faire des règlements, y être autorisé expressément par une disposition législative. C'est pour cela qu'il n'existe dans ces pays que des règlements d'application, alors qu'ailleurs, en France par exemple, la Constitution prévoit, pour schématiser un peu, que tout ce qui est de nature politique relève du Parlement et donc de la loi, et que tout ce qui est de nature administrative relève du pouvoir exécutif, c'est-à-dire du règlement. En conséquence, il existe là-bas des domaines à l'intérieur desquels le gouvernement peut « légiférer» à sa guise. 
Ce que nous appelons règlement se rencontre également sous des dénominations très diverses comme décret, ordonnance, règle, proclamation, arrêté, instruction, directive, etc. II faut prendre garde de ne pas se laisser abuser par l'anarchie terminologique, car ont seuls valeur de loi, la loi et les textes élaborés en vertu d'une disposition expresse de la loi. Les circulaires administratives, les directives, sauf peut-être celles du Conseil du trésor, au Québec, ne sont pas encore de nature législative.

En rapide conclusion sur la hiérarchie des lois, précisons que cette hiérarchie est fondée sur le fait que le règlement doit respecter les principes de la loi, et que la loi doit à son tour respecter les principes de la Constitution.

\section{Les sources du droit}

Les textes que vous avez à traduire ont été rédigés par des juristes formés et fonctionnant dans un système juridique bien caractérisé. La substance des textes dépend d'une part des situations que ces textes entendent régir ou créer et d'autre part de l'état du droit existant. Le rédacteur de lois est amené par exemple à se demander : \& Quelle règle de droit nouvelle dois-je ajouter aux règles composant déjà le système juridique pour agir sur l'état du droit, sur les faits sociaux, ou pour infléchir le comportement social ? $\$$ La nature et le mode de fonctionnement du système juridique dans lequel évolue l'auteur du texte juridique à traduire sont des éléments déterminants de la substance et de la forme de ce texte. $\mathrm{nl}$ est par conséquent essentiel, pour savoir comment on aboutit à ce texte, de connaître même schématiquement les mécanismes de création du droit, les sources du droit.

$\mathrm{Au}$ Québec, le système juridique est composé pour une part de droit britannique, d'autre part de droit français. Le droit britannique est représenté an Québec par le «common law», droit jurisprudentiel, et par le droit criminel (de compétence fédérale). Le droit « français » est représenté par le droit « civil \$, issu du Code civil ou encore Code Napoléon. On oppose le «common law $»$ au système civiliste à cause de leurs profondes différences substantielles et formelles. Cela entraîne pour nous la nécessité de rappeler les sources de chacun de ces sous-systèmes.

\section{Les sources du droit romano-germanique (droit français)}

Je citerai les sources par ordre d'importance décroissante.

a. La loi. La loi est la source principale du droit français, c'est-à-dire que les règles qu'on ajoute les unes après les autres au système juridique existant en droit français sont portées par la loi, votées par le Parlement. Notons qu'en conséquence, la source principale du droit français est écrite.

b. La coutume. Malgré la prééminence de la loi comme source de droit, la coutume, les mours, constituent le fondement de règles, de modes d'organisation sociale qui, au bout d'un certain temps, sont le plus souvent consacrés par la loi. 
c. La jurisprudence. Notons que ce terme a deux acceptions, sensiblement différentes : une acception précise qui ressortit au langage juridique, et une acception générale qui ressortit au langage courant. Dans le langage juridique, on entend par jurisprudence l'ensemble des jugements concordants rendus sur un point de droit donné par les tribunaux de même degré et de même nature. Ainsi, pour qu'il y ait jurisprudence au sens du langage juridique, il faut que, dans des affaires similaires, des tribunaux de même degré aient rendu des décisions concordantes. Dans le langage courant, l'acception du mot jurisprudence est beaucoup plus vague. Il s'agirait cette fois de l'ensemble des décisions ou des opinions rendues par les tribunaux. On entend même parfois qualifier de jurisprudence les tendances influentes de certains organismes qui ne participent pas du domaine judiciaire, Bref, la jurisprudence est l'une des sources du droit français. Il suffit en effet que les tribunaux, de manière concordante, interprètent la loi dans un sens donné, sens qu'on ne lui connaissait pas, pour que dans un premier temps on considère l'interprétation des juges comme étant « la bonne », celle qui s'impose à tous, et que dans un deuxième temps, on consigne dans la loi ou dans un décret d'application l'évolution que les tribunaux auront ainsi fait faire à la loi.

d. La doctrine. On désigne communément par le terme doctrine, sur un point de droit donné, tout ce qui s'est écrit d'important sur ce point de droit. Principalement la doctrine se compose des ouvrages écrits par les chercheurs ou par les professeurs de droit, des articles importants parus sur la matière dans les revues spécialisées, voire parfois dans la presse d'information générale. La doctrine est considérée comme une des sources du droit, puisqu'en fait c'est souvent elle qui, avant même les tribunaux, construit, échafaude des théories d'interprétation et d'application concernant la loi nouvelle. La recherche et l'enseignement universitaires permettent en effet de faire le lien, par exemple, entre des articles de loi ou entre des éléments de jurisprudence, et ainsi de critiquer, d'affiner ou d'augmenter les théories juridiques.

e. Les principes généraux du droit, ou principes supérieurs du droit.

L'importance des principes généraux du droit est, en droit français, considérable. Mais il est difficile à mon avis de les ranger comme source du droit puisqu'ils constituent plus des points de référence, des balises du droit, que des sources.

\section{Les sources du droit d'inspiration britannique} (Toujours par ordre d'importance décroissante)

a. La jurisprudence. La jurisprudence que nous avons définie plus haut a été pendant très longtemps la principale source du droit britannique. $\mathrm{Si}$, à l'heure actuelle, la loi tend à supplanter la jurisprudence comme source directe du droit, la jurisprudence n'en reste pas moins l'agent principal de transformation du droit existant.

b. La loi. Même si, comme on le verra un peu plus loin, la loi est un phénomène anormal pour un esprit britannique, ce mode de création du 
droit envahit petit à petit les systèmes juridiques de type britannique, comme l'Angleterre, le Canada, l'Australie, la Nouvelle-Zélande, etc.

c. La coutume. Pour ceux qui seraient surpris de voir la coutume en troisième position, je rappellerai que, contrairement à ce qui se dit parfois, le droit britannique qu'on désigne parfois par «common law » seulement est surtout un droit jurisprudentiel. Néanmoins, la coutume joue également un rôle dans la détermination de la règle de droit.

d. La raison. Le "common law » a été fondé sur la raison, dissimulée sous la fiction de la coutume générale immémoriale du Royaume. En droit britannique, la raison est ce qui guide le magistrat lorsqu'il rend la justice. Elle s'applique principalement à l'appréciation des faits et des rapports juridiques. Dans les pays de droit écrit, où le droit est principalement législatif, la raison intervient plus spécialement au stade de l'interprétation de la loi. Même dans les pays de droit jurisprudentiel pourtant, on tend à laisser les principes généraux du droit, tirés notamment de décisions judiciaires, supplanter peu à peu la raison comme source du droit. D'autant plus que même chez les tenants de la raison, source du droit, on constate le souci de contenir cet agent dans le cadre d'un système juridique cohérent.

\section{Quelques distinctions entre droit anglais et droit français}

Dans cette partie, j'accorderai une place prépondérante aux sources, à la formation du droit britannique. En fait, tout notre droit, surtout notre droit nouveau au Québec, est influencé par la philosophie et par la formulation du droit britannique et américain. Il est donc important pour maîtriser et parfois contenir cette influence, d'une part de savoir la repérer et, d'autre part, de connaître les tenants et aboutissants culturels et historiques de ce que certains appelleraient la « norme dominante ».

Constatons tout d'abord que ces deux systèmes juridiques sont des produits de l'Histoire. Ils sont néanmoins le produit de deux Histoires différentes.

\section{Le droit français}

Le droit français a été marqué par la faveur qu'on a accordée depuis le XII siècle à la recherche universitaire, à l'enseignement universitaire, à la législation écrite et à la codification. Il a été marqué également par le recours direct à des concepts philosophiques. Par exemple : la justice, conçue comme un but du droit, et la généralité de la règle, conçue comme un des moyens de la justice.

Phénomène beaucoup plus important, la philosophie du droit français admet fort bien que le droit transforme ou même précède la réalité sociale. Le droit est en effet conçu dans ce système comme un outil d'organisation et de transformation sociales. Ainsi, on constate que très tôt le droit français montre la prétention de traiter et d'organiser presque tous les domaines de l'activité humaine. Le juriste français n'a pas hésité à encadrer, à réglementer, à organiser la vie des hommes. 
Le droit français est donc, comme on le constate, marqué par la construction intellectuelle, laquelle permet d'inventer, d'anticiper puis de susciter de nouvelles formes de comportement social. Le droit français est donc un instrument qui permet, non seulement de transmettre, de généraliser, de confirmer une évolution spontanée de la société, mais aussi, d'imposer à tous, par le biais de la loi, un nouvel ordre social directement issu d'une idée abstraite, voire inconnue du plus grand nombre jusqu'alors.

\section{Le droit britannique}

Mise en garde. Certains des propos qui suivent pourraient apparaître par trop négatifs envers le système juridique britannique. Mon but est seulement de faire ressortir pour les besoins de la traduction, les caractéristiques du droit et de la rédaction britanniques qui peuvent égarer le traducteur. Les juristes et rédacteurs britanniques ont leur style, et je n'ai pas l'intention de porter de jugement sur leur style ou leurs méthodes. Il leur appartient de prendre les moyens qu'ils jugent bons pour se faire comprendre.

La rédaction anglaise qui a influencé et influence encore trop souvent le rédacteur francophone a trois caractéristiques :

- d'une part, elle est le produit d'une façon de penser différente de la mentalité des francophones en général

- d'autre part, elle véhicule et diffuse la mentalité britannique qui la soustend

- enfin, elle a nécessairement pour corollaire des principes d'interprétation qui sont également étrangers à l'entendement commun des francophones,

C'est pourquoi, pour permettre au traducteur de repérer les influences et lui éviter de produire une traduction « hors culture», je tente d'exposer les origines à la fois de la mentalité juridique et de la rédaction juridique britanniques. J'en viens à critiquer cette mentalité ou cette rédaction, mais exclusivement dans la mesure où l'on voudrait les plaquer sur des textes destinés aux francophones.

Le droit britannique ou d'inspiration britannique, lui, procède d'une philosophie qui est presque totalement opposée à celle du droit français. Pendant très longtemps, les Anglais ont considéré que le droit n'était pas imposé à la réalité ni dicté par une autorité, mais qu'il se dégageait des faits, et qu'il fallait donc le « découvrir », et non l'« inventer » comme le font les Français. Les Britanniques se sont donc mis, sans grand enthousiasme, sous la pression des nécessités, à découvrir le droit tel qu'il se dégageait des faits.

Pour schématiser à l'extrême, qu'il me soit permis de dire que pour le Britannique, la question est avant tout de résoudre des litiges, et non d'édicter des règles pour un avenir qui, par ailleurs, est incertain. Le Britannique, de toute évidence, ne considère pas le droit législatif comme un type normal de règle juridique. 
Lo Britannique ne crée des règles de droit, à fortiori des règles législatives, que pour pallier l'incapacité partielle et localisée de la société à organiser et gérer ses rapports internes de façon harmonieuse. Autrement dit, il a tendance à attendre de s'apercevoir que quelque chose ne va pas dans un domaine ou dans un autre de l'activité humaine pour intervenir et demander au législateur d'imposer sa solution. C'est pourquoi le droit anglais, que certains considèrent comme infus, implicite dans le corps social, ne s'est " révélé * que lors des procès. Lorsque quelque chose n'allait pas entre deux personnes, entre deux groupes de personnes, on allait voir un juge pour lui dire : «Nous ne sommes pas d'accord. » Le juge appréciait alors les faits du litige, et essayait de dire, souvent en l'absence de texte normatif, quel était le droit.

Il est certain qu'en l'absence de texte, les autres citoyens, surtout s'ils se trouvaient dans une situation à peu près similaire à celle du litige évoqué, étaient tentês de prendre le jugement déjà rendu comme référence pour anticiper la solution de leur propre litige. Ce qui ne faisait l'affaire que d'une des parties en litige, bien sûr.

Néanmoins, pour donner au jugement une portée, une utilité, une autorité dépassant le cadre local et ponctuel du litige déjà tranché, il faut que la solution apportée à ce litige s'impose aux litiges similaires ultérieurs. Et ce, quel que soit le tribunal. C'est notamment pour cette raison que le droit anglais a mis sur pied un système qu'on appelle « la règle du précédent », qui veut qu'un juge soit lié par une solution appliquée précédemment dans des affaires similaires. Cela avait comme avantage, d'une part, comme on l'a vu, la prévisibilité des solutions et, d'autre part, la promotion, la diffusion d'une règle nouvelle en laissant entendre à tous que cette règle s'appliquera dans n'importe quel litige similaire.

On comprend donc, même à ce stade de l'étude, que le droit anglais est un droit d'abord jurisprudentiel, un droit contentieux et, par conséquent, un droit procédurier. Qu'il suffise, à ce propos, de comparer même très rapidement la rédaction d'un traité de droit français et la rédaction d'un traité de droit britannique. On constatera immédiatement que le juriste de droit britannique fonde presque exclusivement les éléments de ses théories sur des décisions judiciaires.

On constate, dans l'Histoire, que le droit anglais s'est donc formé principalement par la compilation des solutions apportées par les tribunaux aux litiges, au fur et à mesure qu'ils se présentaient. Malgré le fait que certains juges aient mêlé à la rédaction de leurs jugements des considérations périphériques à la cause, malgré le fait qu'ils aient eu parfois l'audace, que je me permettrai de qualifier de salutaire, de procéder à des extrapolations, le droit anglais s'est longtemps limité à n'être qu'une compilation de solutions particulières apportées à des problèmes particuliers. La formation du droit britannique n'est donc pas le produit d'une volonté politique d'organisation, mais d'un constat passif et quelque peu partiel de la morale sociale filtrée et interprétée par les magistrats. 
La règle du précédent, qui peut apparaître comme un ferment de cohérence dans le système juridique, puisqu'elle semble pouvoir favoriser la pérennité des solutions, et par conséquent stabiliser l'autorité des principes qui soustendent ces solutions, avait pourtant et comporte toujours d'ailleurs bien des inconvénients. En effet, il est très difficile d'affirmer que deux litiges sont similaires, en raison du temps et de l'espace qui font différer les circonstances.

Par ailleurs, si la règle du précédent favorise la stabilité, elle n'encourage pas toujours la recherche de solutions meilleures. Elle contraint même souvent le magistrat à employer nombre d'astuces pour éviter qu'en plaquant une solution très ancienne sur des faits contemporains, on n'aboutisse à une solution que ne pourrait accepter la morale sociale contemporaine.

On a parfois l'impression, en étudiant les origines du droit britannique, qu'on n'en a développé les moyens qu'avec réticence. Par exemple, si, voici plusieurs siècles, la justice était laissée exclusivement aux tribunaux locaux appliquant les coutumes locales, les tribunaux royaux, première chance d'homogénéisation du droit, ne sont apparus que lorsque les intérêts de la couronne se sont trouvés mis en cause devant les tribunaux locaux. Il fallait en effet que la couronne, pouvoir central, évitât de laisser ses intérêts à la merci d'autorités judiciaires décentralisées. Ce sont les cours royales en fait qui ont institué la règle du précédent comme outil d'uniformisation du droit. Mais au départ, signalons-le, la construction jurisprudentielle de ces cours royales ne s'effectuait que par la transmission orale, d'un juge à l'autre, des données déjà acquises.

Ce n'est qu'au Xmi siècle, selon ce que nous dit le professeur Jean Charlot dans un ouvrage intitulé les Anglais devant la loi, dans la collection U-2, éditions Armand Collin, qu'apparaissent les premiers recueils annuels groupant les arrêts des juges. $\mathrm{Si}$, quelque temps après, on en vient à constituer de véritables recueils de jurisprudence, ces recueils n'en ont pas moins un caractère principalement compilatoire. Il ne s'agit pas encore, loin de là, d'ouvrages pouvant servir de fondement à une véritable théorie du droit. Le « common law » est ainsi constitué pour le principal de précédents auxquels on confère l'autorité de la loi.

La règle du précédent, comme on le soupçonnait un peu plus haut, n'a en fait abouti qu’à figer le droit qui était fondé jusque-là sur la coutume et sur les mœurs.

Le droit, alors dégagé par la jurisprudence des cours royales, n'a pas créé de principes assez généraux pour s'appliquer à toutes les situations. Il a rapidement obligé le juriste de l'époque à recourir à des artifices. Par exemple, on a créé des fictions juridiques, on a sollicité des précédents, on a extrapolé, tout simplement parce qu'il fallait bien, à défaut de principes généraux, que le juge s'appuyât sur quelque chose. En s'éloignant de la sorte de la réalité et de la logique, il est certain qu'on a compromis la prévisibilité des solutions et, par conséquent, la sécurité juridique. 
Les praticiens mêmes du droit se trouvaient désorientés. Le droit et la justice étaient devenus irrésistiblement procéduriers et inadaptés à leur mission. Pour pallier cet inconvénient et redonner à la population un outil propre à rendre une véritable justice, on a développé le système de l'« equity » conçu au départ comme une possibilité de faire appel à l'équité du Roi contre la décision des tribunaux existants. Malheureusement, aux mêmes causes les mêmes effets. La Cour de la Chancellerie, Cour d'《 equity », s'est contentée tout comme l'avaient fait les systèmes précédents, de juger, au fil des procès, par analogie, de compiler les précédents, sans toujours saisir l'occasion d'extrapoler et d'énoncer des principes généraux qui auraient aidé à l'organisation du droit, à l'aménagement de l'état de droit.

Il faut attendre la fin du XIX siècle pour qu'enfin le droit de type législatif commence à installer des principes généraux, et supplante petit à petit les juges dans le rôle de créateur du droit.

Néanmoins, le droit britannique actuel, qui est maintenant largement législatif, garde les stigmates d'un long passé de droit procédurier et de précédents. Ainsi, le législateur britannique répugne-t-il toujours à légiférer à priori et par grands principes. Tout se passe comme si, pour le politicien évoluant en système britannique, le recours à la législation n'était pas, contrairement aux apparences, un pas vers l'organisation globale d'une activité humaine, mais était tout simplement un moyen d'enlever aux tribunaux le pouvoir discrétionnaire d'orienter ou de désorienter les rapports sociaux. Ceci expliquerait que les législateurs des pays de droit britannique n'aient pas vraiment rompu avec les principes, ni avec les méthodes, dégagés de la jurisprudence.

Par-delà la divergence philosophique que nous avons vue plus haut, la divergence méthodologique que nous venons d'examiner longuement est, à mon avis, la source d'une grande partie des problèmes qu'affronte, au Québec comme au Canada, celui qui traduit des textes juridiques.

En effet, vu les deux systèmes juridiques en vigueur au Québec, le fait de passer de l'un à l'autre, par le biais de la traduction, entraîne à passer d'une méthode de pensée à une autre, d'une méthode d'élaboration à une autre et surtout d'une méthode de formulation à une autre. Le traducteur se trouve aux prises avec, d'un côté un droit français qui est systématique et logique, orienté vers des principes abstraits et, de l'autre, un droit anglais qui, pour avoir été construit au hasard des litiges, s'appuie de préférence sur les faits, sur les cas particuliers, sur le concret. Il nous reste à voir les conséquences de tout cela sur la formulation respective des règles de droit en français et en anglais.

\section{CONSÉQUENCES SUR LA FORMULATION DES TEXTES}

Deux systèmes juridiques, aux destins aussi différents que le droit britannique et le droit français, ne pouvaient que produire des textes très distincts quant à leur morphologie, et des principes d'interprétation souvent opposés. 
Tout semble indiquer que le juriste britannique, qui s'est longtemps méfié par exemple du mécanisme conventionnel de la ponctuation, continue de se méfier des réflexes populaires, notamment quant au sens courant et évolutif des mots. Il a également, comme on l'a vu, une aversion profonde pour la généralité. La règle écrite générale et impersonnelle étant, pour lui, un type anormal d'intervention de la puissance publique dans la vie de la société, le juriste britannique en vient même à craindre la généralité des mots pris dans leur sens courant.

C'est une des raisons pour lesquelles les lois britanniques contiennent presque toutes des dispositions qualifiées d'interprétatives et qui, de loin, ressemblent à de la lexicographie. Pour éviter que le justiciable, le praticien et surtout le juge, ne donnent à la loi une portée plus grande que celle que lui destinait le législateur, ce dernier s'exercera à «définir » le plus possible les termes qu'il emploie.

Le législateur se déguise alors en lexicographe, et donne au mot qu'il définit un champ sémantique qui ne correspond pas nécessairement au champ sémantique qu'on lui reconnaît communément. Il étend ou il restreint le sens des mots qu'il emploie, simplement pour faire en sorte que la loi s'applique à tel ensemble de phénomènes, et non à un autre ensemble. Il s'agit d'une entreprise qui est, annonciatrice de problèmes dès le départ, et ceci pour trois raisons.

- Le législateur n'est pas lexicographe. Il ignore le plus souvent, et par conséquent, malmène les principes de la lexicologie.

- Les définitions produites par le législateur entrent en concurrence, d'une part, avec celles que produit le lexicographe et, d'autre part, avec le sens populaire et évolutif des mots.

- La présence de définitions dans la loi crée, à mon avis, immédiatement et parallèlement, une insécurité chez le justiciable, chez le praticien, voire chez le juge. En effet, contrairement au but recherché, l'introduction de définitions dans la loi fait naître dans l'esprit de l'usager l'idée désagréable selon laquelle il devra, tout au long de la lecture, se demander si chacun des termes qu'il lit n'a pas été défini quelque part. En fait, ce procédé fait que le lecteur, quelle que soit sa maîtrise de la langue courante, sent qu'il ne peut plus s'appuyer sur son aptitude ordinaire à comprendre sa langue. Le lecteur des textes juridiques s'attend déjà, la plupart du temps, à rencontrer des mots qu'il ne connaît pas, puisqu'il sait pertinemment qu'il est susceptible de rencontrer des concepts qu'il ignore encore. Ceci est le lot de tous. Mais il est beaucoup plus inquiétant d'avoir constamment à se demander si les mots courants sont employés dans leur sens courant.

Ces difficultés sont autant de défis pour le traducteur qui doit, en plus, se méfier du fait que le même mot est susceptible de recevoir des définitions différentes d'une loi à l'autre.

Pour illustrer les inconvénients de la «définition», prenons l'exemple d'une loi québécoise qui, comme beaucoup d'autres, comporte d'entrée quelques défnitions dont je citerai la plus utile à notre étude. Il s'agit de la Loi sur la sécurité dans les édifices publics, pour laquelle le législateur, empreint des techniques du droit britannique, a ressenti la nécessité de définir les mots «édifices publics ». 
Notons que dans une rédaction propre au droit français, on n'aurait sans doute pas défini dans la loi même ce qu'on entendait par édifice public. Par une méthodologie différente, on aurait choisi d'employer ce mot tel quel dans la loi, laissant à la doctrine et, plus tard, à la jurisprudence, le soin d'estimer s'il est nécessaire de donner à ces mots plus de précision, ou même le cas échéant un sens différent de celui qu'ils ont dans la langue courante. Néanmoins, si le législateur français avait estimé que le concept d'édifice public était assez important en droit pour qu'on lui donnât dans la loi même une définition, il aurait procédé de manière assez abstraite en donnant à ces mots la définition suivante qui n'est de ma part qu'une hypothèse d'école :

Un édifice public est un immeuble dans lequel le public est susceptible de passer ou de séjourner.

Je n'affirme pas ici que telle aurait été exactement la solution adoptée par le législateur de droit français ; je ne cite cette hypothèse que pour indiquer la tendance vers l'abstraction et la généralité qui aurait été la sienne. Voyons maintenant l'exemple de cette définition de type britannique.

Les mots «édifices publics \ employés dans la présente loi désignent les églises, les chapelles, ou les édifices qui servent d'églises ou de chapelles, les monastères, les noviciats, les maisons de retraite, les séminaires, les collèges, les couvents, les maisons d'école, les jardins d'enfance, les garderies, les crèches et ouvroirs, les orphelinats, les patronages, les colonies de vacances, les hôpitaux, les cliniques, les maisons de convalescence ou de repos, les asiles, les refuges, les hôtels, les maisons de logement de dix chambres ou plus, les maisons de rapport de plus de deux étages et de huit logements, les clubs, les cabarets, les cafés-concerts, les music-halls, les cinémas, les théâtres ou les salles utilisées pour des fins similaires, les salles de réunions publiques, de conférences, de divertissements publics, les salles municipales, les édifices utilisés pour les expositions, les foires, les kermesses, les estrades situées sur les champs de course ou utilisées pour des divertissements publics, les arènes de lutte, de boxe, de gouret ou utilisées pour d'autres sports, les édifices de plus de deux étages utilisés comme bureaux, les magasins dont la surface de plancher excède trois mille pieds carrés, les gares de chemin de fer, de tramway, ou d'autobus, les bureaux d'enregistrement, les bibliothèques, musées et bains publics. S.R. 1941, c. 170 , a.2 ; 6 Geo. VI, c. 47 , a.1. (S.R. 1964 , c. 149 , s. I, a. 2)

Cet exemple est intéressant à plusieurs titres. Il illustre ce que je mentionnais plus haut, c'est-à-dire le penchant qu'ont les Britanniques, les juristes en particulier, pour les choses concrètes. En effet, si vous comparez la définition qui est ci-dessus à la définition hypothétique que je proposais pour les mêmes mots, vous apercevrez rapidement que l'anglais tient au plus haut point à toujours décrire concrètement la réalité dont il veut renđre compte, alors que le droit français préférera qualifier cette réalité en prenant pour critère, non pas la description objective et exhaustive des éléments constitutifs de cette réalité, mais par exemple la nature ou la fonction de la réalité en question. Pour résumer, l'anglais décrit la où le français préfère qualifier.

Un deuxième intérêt de cet exemple est de montrer que le juriste de droit britannique semble croire qu'il est possible de décrire une réalité objective par 
l'énumération exhaustive de ses éléments constitutifs. On constate, en effet, que l'énumération des objets que le législateur considère comme des édifices publics se présente comme une énumération exhaustive. Je me permets d'y voir au moins deux défauts potentiels pour l'usager de la loi.

- D'une part, malgré l'ambition qu'a semblé nourrir le législateur d'indiquer de manière exacte à l'usager de la loi ce à quoi elle s'applique lorsqu'elle parle d'édifices publics, il n'a sans doute pas réussi à cerner tous les éléments qu'il aurait voulu viser. En effet, il est presque impossible, selon ce procédé, de ne pas oublier au moins un ou deux éléments importants. Si, de surcroît, le temps, la mémoire ou l'imagination viennent à manquer momentanément au rédacteur, les oublis seront inévitables.

- D'autre part, cette méthode de description de la réalité me semble mal adaptée à la nature du texte de loi. Ce texte est permanent ; ce qui fait que la description limitative restera contenue dans les limites initiales, quelle que soit la durée de validité de cette loi : un an, dix ans, soixante ou cent cinquante ans. Imaginons que, dans une première hypothèse, le législateur ait oublié un élément de la réalité qu'il voulait couvrir. Il faudra reprendre le texte devant le Parlement, avec tous les inconvénients que cela entraîne : inconvénients de retard, inconvénients politiques et même, inconvénients financiers pour le contribuable. Imaginons, dans une deuxième hypothèse, que la liste des édifices publics reste la même pendant plusieurs dizaines d'années. Force nous est de constater que la diversification constante des lieux où le public passe ou séjourne, et où par conséquent sa sécurité doit être imposée par l'Etat, fait qu'il faudrait modifier la loi à chaque fois qu'apparait un nouveau type d'immeuble. En effet, par la force des choses, celui-ci n'aura pu être pris en compte par la liste exhaustive et figée dressée par le législateur.

C'est ce qui s'est produit récemment lorsque le législateur a dâ modifier la définition citée pour ajouter simplement : «les ciné-parcs ».

Par contre, si le juriste a défini l'édifice public comme un immeuble où le public est susceptible de passer ou de séjourner, il n'a pas à craindre d'avoir oublié de cas d'application, sa formule étant assez générale pour que l'usager de la loi, que ce soit le justiciable, le praticien ou le magistrat, puisse aisément vérifier si tel ou tel immeuble correspond aux critères très simples de la définition conçue en termes généraux.

Le titre de certaines lois de type britannique est révélateur. Le législateur n'a pas baptisé la loi dont nous venons de voir un extrait, \&oi sur la sécurité dans les lieux publics ». Il a parlé d'édifices publics. Il semble que lors de l'élaboration, on a d'abord pensé que le problème de la sécurité ne se posait que dans les édifices. On a donc parlé d'édifices. On aurait pu, avant de légiférer, penser que le problème de sécurité qu'on avait constaté dans les édifices pouvait se poser ailleurs. Or, la réticence que le juriste de «common law $\$$ éprouve à légiférer de manière générale a empêché de parler de lieux publics. Cette formule aurait pourtant été plus souple et aurait évité de s'étonner qu'on qualifie d'édifices les ciné-parcs. 
De la même manière, la Loi créant la Commission canadienne du lait aurait dû parler de produits laitiers, car la commission créé a compétence en matière de produits laitiers (produits laitiers qui comprennent, d'après la loi, les sorbets ; soit dit en passant...).

Le juriste britannique, rédacteur du texte que vous aurez à trađuire, comprend très vite qu'il lui sera la plupart du temps impossible de décrire exhaustivement la réalité à laquelle il veut que la loi s'applique. C'est pourquoi il emploie souvent une formule hybride, qui consiste à commencer une énumération comme si elle devait être exhaustive, et à la terminer par une formule telle que « et toute autre chose du même genre $\gg$.

Une fois de plus, nous constatons que si le texte avait été rédigé au départ en français au lieu d'être proposé à votre traduction, le rédacteur francophone aurait sans doute eu le réflexe de commencer par dégager un genre commun aux éléments sur lesquels il veut faire porter les principes de la loi. Ce qui évite d'avoir, une fois de plus, à présenter la réalité selon un découpage objectif et descriptif.

Aux juristes qui prétendent que seul le découpage descriptif et énumératif de la réalité permet de désigner tous les éléments concrets auxquels la loi doit s'appliquer, on peut répondre que, de toute manière, on n'atteint pas le concret absolu avec les mots. En effet, dans la définition donnée en exemple, le mot monastère n'équivaut pas à la réalité matérielle du monastère. Le mot est, dans ce cas, une représentation à un premier niveau d'abstraction de ce qu'est un monastère. La preuve en est que, si l'observation directe et la perception visuelle et tactile de la matérialité d'un monastère permettent d'accéder à une connaissance sans ambiguïté de ce qu'est un monastère, le mot monastère lui-même, comme toutes les représentations abstraites, est susceptible d'interprétations légèrement différentes d'un auditeur à l'autre, d'un lecteur à l'autre ou d'un locuteur à l'autre. Donc, dire que la description exhaustive et élémentaire d'une réalité par les mots qui la désignent équivaut à la concrétisation de cette réalité me semble erroné.

Qu'on ne se méprenne pas, je n'ai d'aucune manière l'intention de juger ou d'influencer les conceptions linguistiques des rédacteurs britanniques, qu'ils soient juristes ou autres. Je me place simplement du point de vue de l'usager de la langue française à qui on demande, non pas de transformer des mots en d'autres mots, non pas seulement de traduire des idées par des idées mais bien plus, de créer à partir d'un texte juridique rédigé en anglais, un texte juridique français qui procède de la même volonté et qui atteigne le même effet.

Il s'agit là de passer directement à l'optique téléologique. Il me semble, après plusieurs années d'étude de ces phénomènes, que pour créer un impact équivalent chez le justiciable anglophone et sur le justiciable francophone, il sera nécessaire de recourir à des moyens logiques ou linguistiques différents. En plus de me fonder sur les constatations ou sur les expériences pratiques que j'ai pu faire dans le domaine de la législation, je prends à témoins les professionnels de la publicité qui ont, depuis quelques années, compris que passer d'une langue à l'autre était souvent insuffisant et qu'il fallait en fait passer d'une culture à l'autre. Le message dans une langue donnée fait très souvent appel à des clichés, à des réflexes cultu- 
rels dont on ne peut transposer l'impact par la seule traduction des mots qui les expriment. Ainsi, pour «vendre » le même produit à deux communautés culturelles différentes, les arguments seront souvent complètement différents.

Je ne vois pas quelle pourrait être l'étrange contrainte qui empêcherait d'adapter les textes juridiques à la culture de ceux à qui ils sont destinés, au lieu de se contenter d'en traduire les mots ou les idées.

Une autre conséquence de la dualité méthodologique que j'ai exposée au début de ces quelques pages, est que le rédacteur britannique ou lié aux méthodes britanniques, cédant comme on l'a vu à sa crainte du générique, voulant dans un deuxième temps recenser et citer toutes les hypothèses d'application de la loi, ne permettra pas de raccourcis ni de sous-entendus dans le cheminement logique de ses idées. Les raccourcis auxquels je fais référence sont ceux qui permettent à un rédacteur francophone de ne pas consigner par écrit, dans le texte juridique par exemple, des éléments ou des détails que le lecteur francophone déduira automatiquement du contexte. Il est des renseignements ou des détails que le rédacteur britannique mentionne en anglais et qui ne sont absolument pas nécessaires dans le texte français. C'est le cas par exemple lorsqu'il s'agit de laisser sous-entendue l'étape intermédiaire d'un raisonnement : lorsque dans le contexte immédiat on aura $\mathrm{A}=\mathrm{C}$, et $\mathrm{B}=\mathrm{C}$, on s'abstiendra de faire constater expressément par la loi que $\mathrm{A}=\mathrm{B}$.

J'aimerais ici citer deux exemples dans le même texte. Il s'agit d'un projet de loi préparé par la Commission de réforme du droit du Canada, en 1975, projet de loi qui visait à modifier le Code criminel en y intégrant un code de la preuve.

On m'avait alors demandé de réviser le texte français. $\AA$ titre d'exemple, le paragraphe 4 de l'article $42 \mathrm{du}$ projet disait en anglais :

There is no privilege under this section :

a) if the purpose of seeking or obtaining the legal services or producing the work or obtaining the information was to enable or aid anyone to commit or plan to commit what the person claiming to hold the privilege knew or reasonably should have known to be a crime or tort ;

Le texte français était le suivant :

Aucun droit au secret ne peut être invoqué en vertu du présent article

a) lorsque les services de l'avocat ont été recherchés ou obtenus, le travail produit ou l'information obtenue dans le but de permettre à quelqu'un de commettre ou de comploter une infraction ou un délit, ou de l'aider à les commettre, et que celui qui réclame le droit au secret a eu connaissance du caractère criminel ou délictuel de l'acte ou a pu avoir raisonnablement connaissance du caractère criminel ou délictuel de l'acte ;

Deux points me semblent importants :

a) Il me semble évident en français que, si le droit au secret ne peut être invoqué du seul fait qu'un individu ait recherché les services d'un avocat dans le but d'aider quelqu'un à commettre une mauvaise action, la personne qui aura obtenu les services d'un avocat pour aider quelqu'un à commettre une mauvaise action ne 
pourra pas non plus invoquer le droit au secret. La personne qui a obtenu, dans le but..., etc., a nécessairement recherché à obtenir. Donc, si pour refuser à une personne le droit au secret, il est suffisant de prouver que cette personne a recherché les services d'un avocat dans le but d'aider quelqu'un à commettre une mauvaise action, il est superflu de mentionner que la personne qui aura obtenu les services d'un avocat dans le but d'aider quelqu'un à commettre cette mauvaise action, n'est pas non plus admissible au droit au secret. Le concept de l'obtention est donc ici une étape intermédiaire dans le cheminement logique des idées. L'anglais a voulu en quelque sorte raconter comment les choses pouvaient se passer, en indiquant tous les cas possibles d'application. Ainsi, il a voulu indiquer que dans le cas où une personne a recherché les services d'un avocat... elle ne pourrait pas invoquer le droit au secret; que, dans un autre cas où la personne aurait obtenu l'aide de l'avocat dans le but... etc., elle ne pourrait pas non plus invoquer le droit au secret. Et ainsi de suite, toutes les situations où le juge pourrait refuser à quelqu'un le droit au secret.

La personne de culture française n'utilise que très rarement cette démarche et préfère assortir la règle de droit de principes généraux d'application, plutôt que de cas particuliers d'application. Une fois de plus, le recours aux principes permet et facilite la déduction, alors que l'énoncé des cas d'application oblige à être exhaustif, à refuser la déduction. C'est pourquoi, dans ce premier exemple, j'ai préconisé qu'on écrive simplement :

Aucun droit au secret ne peut être invoqué en vertu du présent article

a) lorsque les services de l'avocat ont été recherchés, le travail produit ou l'information...

C'est maintenant ainsi que se présente le texte.

b) Dans le même extrait, on trouvait de la part du rédacteur anglais le même souci de préciser tous les cas d'application de la règle. C'est ainsi qu'il prévoyait qu'aucun droit au secret ne pourrait être invoqué par une personne qui aurait eu connaissance du caractère criminel ou délictuel de l'acte, ou qui aurait pu raisonnablement avoir connaissance du caractère criminel ou délictuel de l'acte.

J'ai fait observer que si la loi permettait au juge de refuser le droit au secret à quelqu'un qui $a p u$ raisonnablement avoir connaissance du caractère criminel ou délictuel d'un acte, il serait à plus forte raison fondé de refuser le droit au secret à une personne qui a eu connaissance du caractère délictuel ou criminel de l'acte. Donc, quelle est la condition suffisante pour qu'un juge refuse le droit au secret à une personne qui l'invoque ? La condition suffisante est que cette personne ait pu raisonnablement avoir connaissance du caractère délictuel de l'acte. Pour employer une métaphore boiteuse, si un petit péché, celui d'avoir pu avoir connaissance, suffit à disqualifier la personne, à quoi sert-il d'ajouter qu'un péché plus gros, celui d'avoir eu connaissance, la disqualifierait également? Il s'agit d'un raisonnement non identique, non symétrique, mais parallèle à celui de l'adage qui dit : «Qui peut le plus, peut le moins. 》 Cette fois encore, les autorités francophones de l'organisme se sont rapidement laissées convaincre et, grâce à l'aide énergique et éclairée du professeur Jean-Louis Baudouin, actuellement vice- 
président de la Commission de réforme du droit, le texte français du paragraphe concerné se lit maintenant :

Aucun droit au secret ne peut être invoqué en vertu du présent article

a) lorsque les services de l'avocat ont été recherchés, le travail produit ou l'information obtenue dans le but de permettre à quelqu'un de commettre ou de comploter une infraction ou un délit, ou de l'aider à les commettre, et que celui qui réclame le droit au secret a pu avoir raisonnablement connaissance du caractère criminel ou délictuel de l'acte ;

L'anglais préfère dire :

... what the person claiming to hold the privilege knew or reasonably should have known to be a crime or tort ;

Selon une conception très enracinée, nous aurions pu considérer qu'il y avait une sorte de priorité, de prééminence de la méthodologie anglaise, puisque le texte de départ était un texte anglais. La traduction aurait alors été à mon avis, comme elle l'est trop souvent, un simple habillage avec des mots français d'une règle conçue en anglais. Comme si, pour recourir à une fiction, la loi avait été conçue et rédigée pour des anglophones lisant cette loi en français. Dans l'idéal, il aurait fallu faire un texte anglais et un texte français distincts, non quant à la règle, non quant à la portée, mais quant aux moyens logiques au service de cette portée. C'est ce que j'ai énergiquement soutenu, même s'il s'agissait d'une optique idéaliste en regard des traditions de « fidélité \$ de la traduction juridique, au Québec comme au Canada.

Cette péripétie peut paraître un détail. Elle constitue à mon humble avis une percée dans la muraille de la fidélité traditionnelle, et même une conception un peu plus évoluée de la fidélité, puisqu'elle remet en cause tour à tour le principe du mot à mot, et ce qu'on pourrait appeler la méthode "idée à idée. * Le projet de loi bilingue se présente maintenant de façon radicalement différente de l'anglais au français. Cela va dans le sens d'une libération du traducteur qui alors sert mieux l'intérêt du lecteur et la qualité de la langue. On adapte, en fait, toutes les ressources terminologiques et surtout méthodologiques de la langue d'arrivée au but de l'activité de traduction qui consiste, selon ma conviction, à faire passer un message d'une langue à l'autre, d'une culture à l'autre, en le délivrant dans un premier temps des servitudes culturelles de la langue de départ et, dans un deuxième temps, en mettant au service de sa portée toutes les ressources de la culture d'arrivée.

Toujours dans l'ordre des métaphores boiteuses, je ferai remarquer que lorsque vous passez d'un pays à l'autre, dès la frontière vous vous faites un devoir de respecter les lois, us et coutumes du pays d'arrivée. D'une part dans le but d'éviter les malentendus, d'autre part pour mener avec plus d'harmonie parmi ceux que vous rencontrez l'action que vous êtes venu mener. Il me semble que la situation est un peu parallèle pour le message qui passe d'une langue à une autre, d'une culture à une autre.

Ce que nous avons vu pour la présentation, pour la description des réalités, se retrouve également dans la construction de la phrase. Les textes juridiques 
britanniques comportent des phrases sensiblement différentes de la phrase française traditionnelle. Cela n'est pas seulement dû à une tendance traditionnelle ou naturelle, mais également à une théorie émise par Coode, qui préconise, lui, une structure que je qualifierais de narrative, ou presque dramatique. Coode conseille de commencer la phrase en plantant le décor, c'est-à-dire en exposant d'abord les circonstances d'application de la règle que porte la phrase. Il y aurait ainsi les circonstances, et, dans l'idéal, à la fin de la phrase, il y aurait ce qu'il appelle le « legal subject » et enfin la « legal action».

Dans la pratique, les choses se compliquent. La théorie de Coode n'empêcherait pas à priori qu'on mette, comme je le préconise, le principe de la règle énoncée dans la proposition principale, et les circonstances d'application dans des propositions subordonnées. Et d'après ce que l'on peut constater, dans les textes juridiques anglais, le principe, la règle, ne se situe pas systématiquement dans la proposition principale.

Or, je crois qu'il est nécessaire de conserver, en français, quelles que soient les habitudes anglaises, une structure de la phrase qui mette systématiquement en relief l'idée principale et relègue les idées secondaires au rang des propositions circonstancielles ou des incises.

Il faut également remarquer l'attirance des rédacteurs juridiques britanniques pour les phrases très longues, assez complexes et peu ponctuées. Voici un exemple qui rassemble les deux caractéristiques citées, c'est-à-dire la structure anglaise selon Coode et la longueur de la phrase, attribuable paraît-il à une apparition tardive de règles claires en matière de ponctuation. L'exemple qui suit pourra vous parâ̂tre à priori mal choisi ou surprenant, car il s'agit d'un texte récent, rédigé en français. Il illustre par osmose les caractéristiques de la rédaction britannique.

L'article 24 du Règlement 77-486 du 26 août 1977 prévoit que :

Si l'enfant recevait légalement l'enseignement en anglais en 1976-1977 dans une autre commission scolaire ou institution, l'inscription peut être faite, sous réserve d'une décision contraire de la personne désignée par le ministre, si le directeur général de la commission scolaire ou le directeur de l'institution qui dispensait l'enseignement en 1976-1977 peut en attester auprès de celui où se fait la demande ou si la commission scolaire ou l'institution obtient de la personne désignée par le ministre une attestation écrite à l'effet que le nom de l'enfant apparaît sur la liste des élèves inscrits à l'enseignement en anglais en 1976-1977 émise par le ministère de l'Education.

Cet article, pourtant rédigé en français, suit à peu près les prescriptions de Coode qui veut qu'on commence la phrase par des propositions circonstancielles. $\mathrm{Si}$ nous procédons à une analyse logique de cette très longue phrase, nous trouvons la proposition principale à la deuxième ligne : «L'inscription peut être faite ». Le reste n'est que propositions circonstancielles ou incises, qui expriment des conditions à l'inscription. Notons au passage que ces conditions qui, d'après le sens de l'article, sont sur le même plan, semblent ne pas l'être du fait d'une part qu'elles se situent avant et après la proposition principale, et d'autre part qu'elles sont introduites de manière différente. En effet, certaines de ces conditions sont 
introduites par «si » : «Si l'enfant recevait légalement l'enseignement en anglais... sous réserve d'une décision contraire... si le directeur général de la commission scolaire... si la commission scolaire ou l'institution obtient... de la personne désignée par le ministre... la liste... émise par le ministère de l'Éducation ». Lorsque les conditions sont introduites par "si », on peut les repérer aisément. Néanmoins, lorsque à l'intérieur de l'énoncé d'une condition, on exige que l'attestation soit délivrée par la personne désignée par le ministre, il s'agit d'une condition déguisée, puisqu'il n'est pas seulement nécessaire d'obtenir l'attestation; encore faut-il qu'elle soit délivrée par la personne désignée par le ministre. Le même raisonnement sommaire peut s'appliquer à la qualification de la liste qui, elle, doit être émise par le ministère de l'Éducation.

Mais revenons aux choses fondamentales. Il m'apparaît que le but de l'article n'était pas de dire, comme le dit la proposition principale, que l'inscription peut être faite. Tout le monde s'en doute, puisque si on ne pouvait pas faire l'inscription, on n'y mettrait pas autant de conditions. Le but de l'article était en fait de signaler qu'il y avait des conditions à l'inscription, et d'énoncer ces conditions. II me semble qu'alors la proposition principale aurait dû être, en français, le lieu privilégié où indiquer qu'il $\mathrm{y}$ a des conditions à l'inscription et de reléguer ces conditions à une énumération. On aurait pu dire :

Pour qu'un enfant qui recevait légalement l'enseignement en anglais en 1976-1977 puisse continuer de le suivre dans une autre institution ou dans une autre commission scolaire, plusieurs conditions sont nécessaires.

1. Il faut que...

2. Il faut que...

3. Etc.

Ainsi, la proposition principale contient l'idée principale de la phrase. La phrase sera un peu moins longue, et les conditions seront d'une part mieux distinguées, d'autre part mises sur le même plan.

La longueur des phrases, elle, est un domaine où votre libre arbitre et votre talent vous permettront d'être infidèle à la ponctuation anglaise pour être mieux fidèle à la stylistique française. Dans le domaine juridique où les textes de loi, par exemple, portent la norme sociale, s'imposent à tous, confèrent des obligations, garantissent des libertés individuelles, il est essentiel que le justiciable puisse accéder au sens, quel que soit son degré d'instruction. Que le législateur anglais ou celui des pays de droit britannique juge bon de se méfier des techniques de la ponctuation, qu'il estime plus opportun de présenter aux anglophones des phrases longues, d'apparence sinueuse, ne doit pas nous amener à porter systématiquement un jugement de qualité sur la base d'une comparaison avec le français. Les anglophones écrivent pour les anglophones et, à mon avis, il n'appartient qu'à eux de critiquer et de redresser ce qui pourrait leur apparaître comme des défauts dans leurs textes. Sans doute ont-ils une plus grande habitude du cheminement dans les phrases longues et complexes.

Qu'il me soit permis, à ce propos, de vous livrer une association d'idées qui m'est souvent venue en pensant à ce phénomène : la personne de culture ou de 
formation anglaise, britannique, fondamentalement éprise d'individualisme et de liberté individuelle, plus que réticente devant la symétrie et les autres sous-produits du formalisme, n'est-elle pas plus habituée que nous, francophones, à retrouver son chemin dans des phrases dont les éléments se succèdent en fonction de la chronologie des événements dont ils rendent compte? Les phrases anglaises me font parfois penser à des jardins à l'anglaise. Toutefois, s'il est vrai que le promeneur est charmé par le jardin à l'anglaise qui, malgré la variété et la richesse des essences, semble être une improvisation de la nature, celui qui doit traverser un jardin rapidement et sans se perdre préférera le jardin à la française dont les alignements et la symétrie permettent au moins de trouver facilement le chemin le plus direct.

J'ai toujours été surpris de la propension de la langue juridique à la «tournure négative » et à la double négation. Exemple :

La personnalité juridique ne peut être conférée à moins que la raison sociale adoptée ne soit en langue française. (Article 30 de la Loi sur la langue officielle, dite « loi 22 »)

Nous avons tous hérité de nos instituteurs la règle selon laquelle l'idée principale de la phrase doit de préférence être située dans la proposition principale. Dans l'exemple cité, la proposition principale est :

La personnalité juridique ne peut être conférée...

Il est clair que le principe porté par l'article n'était pas de priver les compagnies de personnalité juridique. Il s'agissait tout simplement d'exiger qu'on rédigeât les raisons sociales en français. On aurait pu écrire :

Une compagnie doit avoir une raison sociale en langue française pour obtenir la personnalité juridique.

En plus de mettre l'accent, dans la proposition principale, sur l'effet véritable de l'article, cette formulation a pour avantage d'éviter les inconvénients de ce que je qualifie de tournure négative. En effet, je suis certain que le fait de dire dans la proposition principale d'une phrase que les compagnies ne peuvent s'enregistrer au Québec crée un impact négatif sur l'inconscient du lecteur, même si ce dernier paraît décoder sans difficulté le message présenté de façon indirecte. La fréquence de ces tournures négatives ne peut manquer néanmoins de donner aux textes un arrière-goût de répression. En toute inutilité d'ailleurs. La norme sociale est déjà contraignante, et la répression détestable. Essayons de n'y recourir que dans les cas où cela est inévitable, c'est-à-dire dans le domaine pénal. tention.

Quant à la double négation, elle est sans doute le produit d'une faute d'inat-

L'article 14 de la loi canadienne concernant le Conseil des ports nationaux dispose que :

The Governor in Council may make by-laws not inconsistent with the provisions of this Act...

Le Gouverneur en conseil peut établir des règlements non incompatibles avec les dispositions de la présente loi... 
Deux remarques : court ?

- Pourquoi \& non incompatibles \&? Pourquoi pas \& compatibles * tout

- La mention de la compatibilité du règlement est inutile car, par principe, le règlement ne peut sans autorisation de la loi aller à l'encontre de cette dernière.

Enfin, un dernier exemple de tournure négative. Il est connu, en français comme en mathématiques, que deux négations s'annulent ou équivalent à une affirmation $(-\times-=+)$. Le cas est-il identique en matière d'exceptions? Exemple : la loi créant une commission canadienne du lait prévoit dans son article 14 que :

Toutes les dépenses pour traitement, frais de voyage et d'administration, à l'exclusion de celles qui, de l'avis du Ministre, sont directement imputables aux mesures prises par la Commission pour stabiliser les prix de quelque produit laitier, doivent être payées sur les crédits affectés à cette fin par le Parlement.

L'article 15 (3) dispose que :

Doivent être payées sur le Fonds du revenu consolidé... toutes les dépenses ressortissant à la présente loi, sauf celles payées conformément à l'article 14 ...

- Sauf >, ici, renvoie à un ensemble (l'article 14) qui lui-même contient une exclusion. Comment interpréter cette somme algébrique de retranchements? Le problème est de savoir en quoi consistent les sommes qui doivent être payées sur le Fonds du revenu consolidé : Qui doit payer quoi?

Les mathématiques résoudraient-elles le problème?

Soit « a : les dépenses pour traitements, frais de voyages et d'administration ; Soit $\&$ b : les dépenses pour traitement, frais de voyage et d'administration qui sont directement imputables aux mesures prises par la Commission pour stabiliser le prix d'un produit laitier ;

Soit $\ll \mathrm{c}\rangle:$ les dépenses ressortissant à la présente loi ;

Soit $\& \mathrm{~d}$ : : les dépenses ressortissant à la présente loi et payées conformément à l'article 14 ;

Soit * e : les dépenses qui doivent être payées (article 15-3) sur le Fonds du revenu consolidé.

Partant du fait que : $d=a-b$

nous avons

$$
\begin{aligned}
& e=c-d \\
& \text { donc : } e=c-(a-b) \\
& \text { donc }: e=c-a+b
\end{aligned}
$$

L'équation est le seul moyen, bien qu'aléatoire dans ce cas, d'arriver à savoir que les sommes représentées par $* b$ * seront payées à même le Fonds du revenu consolidé. Un cheminement bien ardu vers la compréhension... Evitons donc de mettre " en série s les négations ou les exceptions. 
Bien d'autres aspects de la traduction juridique seraient sans doute intéressants à traiter ici. Outre la propension britannique à la voix passive qui, à mon avis, a souvent l'inconvénient de mal révéler qui fait l'action, la propension des rédacteurs britanniques et français à s'exprimer à la forme négative, par exemple à exprimer une interdiction là où il serait suffisant d'exprimer une obligation ; la propension des juristes à choisir parmi les synonymes les mots les moins courants ; ou encore, sans être exhaustif, la mauvaise habitude de considérer qu'un texte juridique est nécessairement hermétique dont le sens est réservé aux oracles du droit que sont les juristes, d'autres aspects pourraient encore retenir votre bienveillante attention, comme l'absence de dictionnaire juridique, utilement orienté vers le droit québécois, le droit canadien. Enfin, est-il possible de traduire du droit, non seulement d'une langue à l'autre mais d'un système à l'autre en conservant aux règles traduites la même portée?

Sur ce dernier point, j'aimerais rappeler qu'à mon avis l'ensemble des règles juridiques que se donne une communauté est le reflet de la conception que cette communauté se fait de l'organisation de ses rapports internes. Ne serait-ce qu'à cet égard, je suis convaincu que traduire des règles juridiques n'est pas tellement traduire des mots, traduire des idées, mais que c'est tout simplement, comme on le fait actuellement, «importer » des méthodes d'organisation sociale étrangères. C'est pourquoi, en dehors de toutes les conséquences politiques qu'on pourrait en tirer, on aboutira fatalement à la conclusion selon laquelle la rédaction parallèle est préférable à la traduction. La rédaction parallèle n'est d'ailleurs qu'un palliatif et ne résout pas vraiment la difficulté de plus en plus évidente de produire des lois qui respectent deux cultures à la fois.

\section{LE TRADUCTEUR ET LE JURISTE}

Je terminerai cet exposé par quelques considérations sur les rapports entre traducteur et juriste.

J'ai souvent remarqué, depuis que j'ai le plaisir de côtoyer des traductrices et traducteurs, que vous avez su élever l'art du compromis au niveau de la vertu scientifique. Néanmoins, et ceci ne cesse de m'étonner, je constate, qu'on me pardonne ma franchise, que le traducteur a trop souvent tendance à considérer sa profession comme un simple accessoire à l'ensemble des professions dites spécialisées. D'une part, je pense que votre profession n'est pas accessoire mais complémentaire... comme toutes les professions. Aucune formation professionnelle, si longue et si ardue soit-elle, ne prépare à la compétence universelle. Chacune des professions n'est donc point accessoire à l'ensemble des autres, mais est, comme chacune des autres, un complément nécessaire. Que feraient quinze médecins isolés sur une île déserte ? Seraient-ils capables d'assurer même leur propre santé, habitués qu'ils sont à l'aide d'un personnel et d'un équipement spécialisés ? Que feraient quinze avocats ou quinze traducteurs dans les mêmes circonstances ? Le médecin, l'électricien, le juriste ont chacun reçu une formation qui leur permet de manier des techniques particulières. De la même manière, le traducteur a été formé pour être capable, grâce à des techniques nombreuses et complexes, de faire passer un message d'une langue à une autre, d'une culture à une autre. Quels 
que soient les critères ou les influences qui vous porteraient à penser que votre profession n'est pas sur le même plan que celles des autres spécialistes, je ne saurais trop vous conseiller, dans un premier temps, de revoir votre analyse et, dans un second temps, de commencer par vous considérer vous-mêmes comme des spécialistes parmi d'autres, au lieu d'attendre l'effet hypothétique que pourrait avoir, sur votre cote de prestige, une reconnaissance officielle de votre art par l'Office des professions.

Parlons franc. Il y a bien sûr dans mes propos le côté emporte-pièce d'une optique militante. Il est certain que le statu quo prudent compense les petites frustrations professionnelles par la stabilité d'emploi. Loin de mépriser cet état d'esprit, qui est le lot de la presque totalité des prestateurs de services, je n'en pense pas moins que vous avez à votre portée les moyens de faire mieux connaître et reconnaître, non seulement vos aptitudes, vos capacités, mais par là même la meilleure utilisation que d'autres spécialistes pourraient faire de vos services.

La profession juridique est marquée par une attitude généralement conservatrice et orientée vers le concept bien commode de sécurité juridique. Le juriste de formation britannique, qui a quelque raison de craindre l'insécurité juridique, va souvent opposer aux innovations de formulation, particulièrement à une révision importante de la notion de fidélité dans la traduction, les impératifs premiers de sécurité juridique. Il aura tendance, à juste titre, à ne point admettre qu'une * fantaisie $\gg$ de formulation affecte le fond du texte. Il est toutefois encore trop souvent marqué par la vieille dualité fond-forme. Depuis quelques années, j'affirme que cette vieille division n'est pas commode, car personne, à ce que $\mathrm{j}$ 'ai constaté, n'est capable de dire où s'arrête la forme et où commence le fond. Je propose que cette vieille division, que certains croient possible d'appliquer à la lettre, laisse la place à une indication de tendance sur la nature et les rapports de trois éléments : le fond, la structure et le style. Je n'avancerai pas plus dans cette théorie qui pourrait à elle seule faire l'objet d'un autre article.

Peut-on répondre à l'argument de la sécurité juridique? Oui. En effet, il arrive souvent qu'une traduction littérale, ce n'est pas à vous que je l'apprendrai, livre en français un message sensiblement différent du message initial en anglais. La portée du texte d'arrivée est par conséquent différente de la portée du texte de départ, ce qui compromet l'égalité des deux textes et, par conséquent, la sécurité juridique.

Dans un autre ordre d'idée, un texte confus est toujours de nature à compromettre la sécurité juridique. La fidélité du traducteur doit-elle le conduire à simplement traduire la confusion par la confusion, ou doit-elle l'amener à signaler à celui qui commandite le travail l'incohérence apparente du texte de départ ? Dans une telle situation, à ce que j'ai remarqué, certains traducteurs partent du principe que si le texte est confus, c'est qu'il est sans doute technique, et que s'il est technique, il est normal qu'on ne le comprenne pas. Ce qui porte ces traducteurs à ne pas remettre en question le sens du texte de départ et à le rendre tant bien que mal en français. Trois raisons, au moins, me poussent à déconseiller cette attitude. 
- D'une part, la majorité des textes juridiques sont destinés à être compris par le plus grand nombre. Si vous ne les comprenez pas vous-mêmes qui n'êtes pas juristes de formation, il y a des chances que les autres non-juristes ne les comprennent pas eux-mêmes.

— La technicité n'est à mon avis jamais une excuse à l'obscurité. Il est toujours possible d'exprimer un argument clairement.

- Si vous ne faites pas remarquer l'ambiguïté ou l'incohérence dont on s'apercevra tôt ou tard, la personne qui vous commandite ce travail peut en déduire que vous ne l'avez pas vous-mêmes remarquée et que, par conséquent, votre acuité de perception des phénomènes linguistiques est faible. Il est désagréable parfois de se faire montrer qu'on a commis une incohérence, mais j'imagine qu'il est encore plus désagréable pour un traducteur de se faire dire qu'il a laissé passer une incongruité alors qu'il l'avait, en fait, repérée, sans oser la faire remarquer.

Le juriste, comme bien des spécialistes, n'éprouve aucune réticence, au contraire, à guider son client parmi les méandres de la technique qu'il maîtrise. C'est pour cela que le client recourt à ses services. Il me semble que celui qui commandite votre traduction est en droit d'attendre, parfois malgré lui, que vous le guidiez vers un texte d'arrivée d'une excellente qualité.

Selon une idée reçue que j'ai également constatée dans le milieu de la traduction, il existerait une infériorité fondamentale du traducteur par rapport au juriste. Elle résiderait, paraitt-il, dans le fait que le traducteur ne connaît pas le droit, alors que le juriste connaît sa langue. Il s'agit d'une conception regrettable qui résulte d'une analyse pour le moins sommaire. En effet, si le juriste connaît sa langue, il n'a pas suivi la plupart du temps les trois ans de cours universitaire pendant lesquels le traducteur a acquis plus qu'une connaissance de sa langue : une connaissance des techniques les plus affinées en matière de traduction. La connaissance que le juriste a de la traduction est une connaissance en grande partie infuse. Cette connaissance infuse de la traduction peut sans doute être comparée à votre connaissance infuse du droit, ou de vos droits, de ce que vous avez le droit de faire, de ce que vous n'avez pas le droit de faire dans votre comportement social. J'estime, par conséquent, que vous êtes sur le même plan. Le juriste n'acceptera sans doute pas que vous contestiez le fondement juridique de son argumentation. De la même manière, est-il adroit de laisser celui qui commandite votre traduction vous imposer ses conceptions, le plus souvent très incomplètes, de la traduction?

Sans aller jusqu'au malentendu ni jusqu'à la rupture des relations professionnelles, il vous est toujours possible de faire valoir vos arguments.

Le fait d'approuver un produit fini qui n'est pas digne de votre niveau peut être un compromis habile dans l'immédiat, mais pardonnez-moi de le penser, n'est ni honnête ni rentable pour l'avenir. C'est un peu comme si un médecin laissait sans protester ses patients appliquer à leur guise les thérapies qu'il a prescrites, simplement pour ne pas les contrarier. Le gain en popularité est immédiat, mais ne saurait se maintenir très longtemps : les malades s'aperçoivent dans un premier temps qu'ils ne guérissent pas et, dans un deuxième temps, changent 
de médecin, s'ils ne sont pas déjà morts. En définitive, et paradoxalement; c'est pour offrir a votre client un service digne de votre profession que vous serez amenés à maintenir d'une manière très énergique, très haute et selon vos critères, la qualité des textes que vous produisez.

À condition de réagir rapidement, d'être persuasifs et patients, vous avez toutes les raisons d'être optimistes quant au développement des rapports entre les traducteurs et les spécialistes qui commanditent vos traductions. L'autocensure ne fera rien pour votre popularité ni pour le prestige de votre profession. Il me semble qu'à force de présenter, au juriste comme aux autres spécialistes, des textes d'une grande précision, d'une grande simplicité et le plus conformes possible à la culture d'arrivée, vos interlocuteurs les plus récalcitrants finiront, d'une part, par savoir ce qu'est la qualité et, d'autre part, se fatigueront de se battre contre elle.

\section{CONCLUSION}

Le domaine de la traduction juridique subira l'influence de l'évolution prochaine et fondamentale des techniques de rédaction des textes juridiques. Les effets conjugués de l'amélioration de la rédaction juridique en français et de la disparition depuis le 26 août 1977 du bilinguisme officiel dans la législation font que les problèmes d'adéquation culturelle qui se posaient dans les textes juridiques jusqu'à très récemment, de l'anglais au français, risquent cette fois d'être renversés, puisque de plus en plus, c'est du français à l'anglais qu'on va traduire les textes juridiques. De plus, les textes français qui appelleront une traduction en anglais seront de moins en moins des textes conçus à l'anglaise avec des mots français. Soyons vigilants et essayons de faire en sorte que les inconvénients qui accompagnaient, pour la culture française, la traduction de textes anglais, ne se retournent pas contre la culture des anglo-québécois.

Les problèmes qui accompagnent la transplantation de la norme juridique d'une culture dans une autre culture, problèmes dont nous n'avons fait qu'effleurer la masse, ne sont pas près de recevoir une solution juridique ou linguistique. Je suis convaincu qu'à moins de réaménager les rapports, apparemment inextricables, des deux systèmes de droit en vigueur au Canada, notamment sur le plan de la rédaction et de l'interprétation des lois, il n'est pas possible d'envisager la fin prochaine des pratiques de base de la traduction juridique.

N'oublions pas qu'au Canada, il y a onze parlements, onze gouvernements, onze systèmes judiciaires, deux cultures principales, deux langues principales, au moins quatre sources de droit législatif par province (Assemblée nationale, réglementation provinciale, Chambre des communes, réglementation fédérale). $\mathrm{Au}$ Québec, deux systèmes juridiques coexistent... J'admire et j'envie ceux qui peuvent imaginer d'une part que les divers systèmes juridiques évoluent tout en gardant chacun ses caractères et d'autre part qu'on pourra un jour proposer au traducteur des « passerelles s suffisamment sûres pour qu'il puisse transporter le message juridique intact d'une rive culturelle à l'autre, sans pour cela avoir à se battre quotidiennement pour sa langue et pour sa profession.

Michel SPARER 


\section{BIBLIOGRAPHIE}

BLACK, H.C., Black's Law Dictionary, St. Paul (Minn.), West Publishing Co., 1968.

GUILLIEN, Vincent, Lexique des termes juridiques, Paris, Dalloz, 1978.

DICK, Robert C., Legal Drafting, Toronto, Carswell, 1972.

PIGEON, L.P., Rédaction et interprétation des lois, Québec, Editeur officiel du Québec, 1965. SOURIOUX, P. et P. LERAT, le Langage du droit, Paris, P.U.F., 1975.

SPARER, Michel, Propos sur la rédaction des lois, Colloque international organisé et animé par $\rightarrow$, Conseil de la Iangue française, Québec, Editeur officiel du Québec, 1978. 